\title{
Supreme Courts and Courts of Cassation
}

\author{
Citation for published version (APA):
}

Bravo Hurtado, P. (2018). Supreme Courts and Courts of Cassation: A diagonal symmetry: Comparative law study of the US, England, France and Italy in civil procedure. [Doctoral Thesis, Maastricht University]. Maastricht University. https://doi.org/10.26481/dis.20180911pbh

\section{Document status and date:}

Published: 01/01/2018

DOI:

10.26481/dis.20180911pbh

Document Version:

Publisher's PDF, also known as Version of record

\section{Please check the document version of this publication:}

- A submitted manuscript is the version of the article upon submission and before peer-review. There can be important differences between the submitted version and the official published version of record.

People interested in the research are advised to contact the author for the final version of the publication, or visit the DOI to the publisher's website.

- The final author version and the galley proof are versions of the publication after peer review.

- The final published version features the final layout of the paper including the volume, issue and page numbers.

Link to publication

\footnotetext{
General rights rights.

- You may freely distribute the URL identifying the publication in the public portal. please follow below link for the End User Agreement:

www.umlib.nl/taverne-license

Take down policy

If you believe that this document breaches copyright please contact us at:

repository@maastrichtuniversity.nl

providing details and we will investigate your claim.
}

Copyright and moral rights for the publications made accessible in the public portal are retained by the authors and/or other copyright owners and it is a condition of accessing publications that users recognise and abide by the legal requirements associated with these

- Users may download and print one copy of any publication from the public portal for the purpose of private study or research.

- You may not further distribute the material or use it for any profit-making activity or commercial gain

If the publication is distributed under the terms of Article $25 \mathrm{fa}$ of the Dutch Copyright Act, indicated by the "Taverne" license above, 


\section{SUMMARIES}

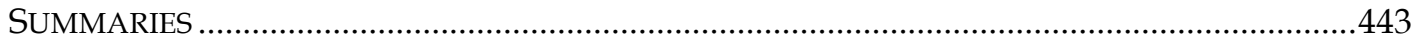

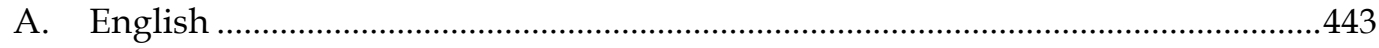

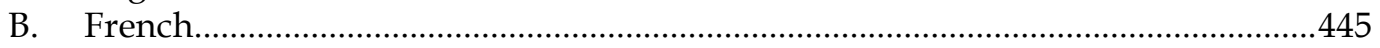

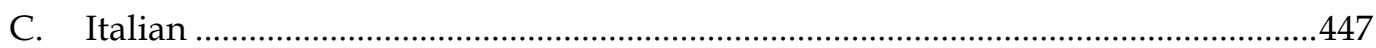

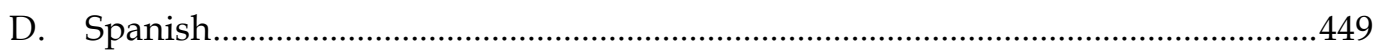

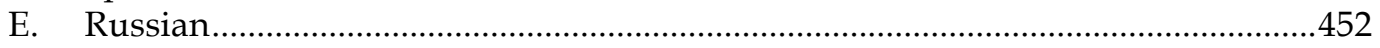

\section{A. English}

This dissertation, titled 'Supreme Courts and Courts of Cassation: A Diagonal Symmetry', is a comparative study of the judicial functions of the courts of last resort in civil matters of two pairs of jurisdictions that belong to the common law (US and England) and the civil law (France and Italy). The methodology consists of the comparison of eight functional attributes of these courts - i.e., scope of review, effects of the judgment, decision exposure or publication, opinion style, caseload, panel composition, total size and preliminary screening (access filters) - but for the purposes of this Summary, let us concentrate on two of these attributes: the effects of the judgments and the opinion style. The main findings challenge the orthodox view about the divide between the common law and the civil law as regards their (apparently) different judicial functions. The research demonstrates that, in reality, both pairs of jurisdictions have courts with similar judicial functions, but located at different levels of the court hierarchy, as follows. 
Figure I : Summary - A Diagonal Symmetry

US + ENGLAND $\quad$ FRANCE + ITALY

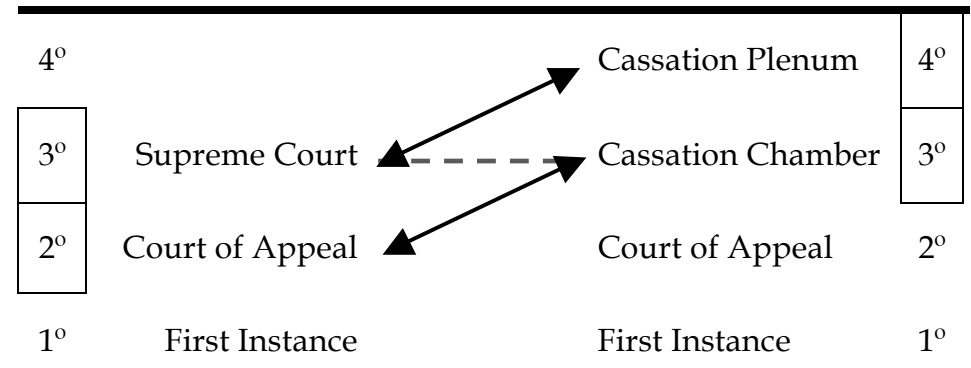

According to the orthodox view of comparative law, common law jurisdictions such as the US and England are characterised by, among other aspects, decisions that have a strong force to change the decision criteria of future cases (binding precedents) and judgments that include dissenting opinions. Civil law jurisdictions such as France and Italy - the orthodox view continues - are characterised, quite the contrary, by judicial decisions of a weaker force, because only a trend of several judgments in the same direction may change the criteria of future cases (jurisprudence constante) and expressing dissenting opinions among judges is not allowed.

That orthodox divide between the common law and the civil law certainly holds true from a 'horizontal' perspective - that is to say, when comparing courts located at the same $3^{\text {rd }}$ level of each pair of jurisdictions: the supreme courts of the US and UK vis-à-vis the 'chambers' of the courts of cassation of France and Italy. Indeed, the judgments of the US and UK supreme courts have a strong binding force and dissenting opinions are rather common. On the other hand, the decisions delivered by the ordinary chambers of the French and Italian cassation courts do not enjoy an equivalent binding force and their judgments omit any kind of dissenting opinion.

The same orthodox divide, however, does not hold true if we change our perspective from a horizontal one to a 'diagonal' one, comparing courts at different hierarchical levels. As this dissertation demonstrates through the comparison of the eight attributes relevant to the courts' functioning, France and Italy also exhibit judicial organs that perform similar functions as the US and UK supreme courts, but at a hidden higher level. The strong force of decisions in guiding future cases and (rudimentary forms of) dissenting opinions can be found not in the ordinary chambers but in the 'plenary sessions' of the French and Italian cassation courts. Therefore, the 'common law' judicial function found at the $3^{\text {rd }}$ level of the US and UK supreme courts is also present - however, phrased in a different manner and not always explicitly admitted - at the hidden $4^{\text {th }}$ level of the cassation plenum in France and Italy.

Additionally, this dissertation demonstrates that the judicial functions of the ordinary chambers in the French and Italian cassation courts can also be found in the common law jurisdictions of the US and England, but at a lower court level. As a reaction to the case overload crisis, the US and English intermediate appellate courts now deliver judgments that have a much weaker precedential force (comparable to the jurisprudence constante 
system), judgments in which dissenting opinions are becoming impractical and exceptional. Therefore, the judicial function that according to the orthodox view is proper of the civil law is becoming more and more apparent at the $2^{\text {nd }}$ court level in the US and England as well.

These findings - called the 'Diagonal Symmetry' thesis - are relevant for several reasons. First, the diagonal symmetry invites us to reformulate the divide between the common law and the civil law. From now on, the real divide is not in the distinctive judicial functions of each legal tradition, but at the court level in which the equivalent judicial functions are present. Second, the diagonal symmetry changes the relevant question that further comparative law studies should answer. From now on, the relevant question is not why the common law and the civil law have different judicial functions, but why civil law jurisdictions (such as France and Italy) perform at higher court levels the functions that common law jurisdictions (such as the US and England) perform at lower court levels. Third, the diagonal symmetry is important for policy-makers because it demonstrates that the relevant point when designing a judicial reform is not about which functions they want the courts to perform, but at which level of the court system they want those functions to be performed. Finally, the diagonal symmetry may facilitate the international circulation of judicial models - such as the introduction of binding precedents in civil law jurisdictions - because it demonstrates that judicial functions that are thought to be proper of foreign legal traditions are, in reality, also present in domestic jurisdictions. Therefore, the so-called 'transplants' of these kinds of judicial functions should not be seen as the introduction of foreign institutions but as the relocation of domestic judicial functions that were previously performed at different court levels. In that sense, the findings of this dissertation will be of invaluable help in promoting these improvements in legal education, comparative research and judicial reforms that follow from the diagonal symmetry thesis.

\section{B. French}

Cette thèse de doctorat intitulée 'Cour suprême et cour de cassation: une symétrie diagonale' [Supreme Courts and Courts of Cassation: A Diagonal Symmetry], est une étude comparative des fonctions judiciaires des tribunaux de dernière instance en matières civiles dans deux paires de juridictions relevant de la common law (les Etats-Unis et l'Angleterre) et du civil law ou de la tradition romano-canonique (la France et l'Italie). La méthodologie utilisée consiste à comparer huit attributions fonctionnelles des cours - i.e., le champ de révision, les effets des décisions, la publicité des décisions, le nombre d'affaires, évaluation préalable (ou filtre d'accès), la composition des formations de jugement, l'étendue totale et le style des décisions. Cependant, aux fins du présent résumé, nous allons nous concentrer sur deux des attributions fonctionnelles, à savoir, les effets et le style des décisions de justice. La principale conclusion de cette étude remet en question la vision orthodoxe de la division entre la common law et la civil law à propos des (supposées) différences dans leurs fonctions judiciaires. Cette recherche montre, qu'en réalité, les deux paires de juridictions ont des cours avec des fonctions judiciaires semblables, mais qui sont situées à des niveaux hiérarchiques différents, comme il en suit : 
Schéma I : Résumé - Une symétrie diagonale

\begin{tabular}{|c|c|c|c|}
\hline & $\begin{array}{r}\text { ETATS-UNIS + } \\
\text { ANGLETERRE }\end{array}$ & $\begin{array}{l}\text { FRANCE + } \\
\text { ITALIE }\end{array}$ & \\
\hline $4^{\circ}$ & & $\begin{array}{l}\text { Formation } \\
\text { plénière }\end{array}$ & $4^{\mathrm{o}}$ \\
\hline $3^{\circ}$ & $\begin{array}{l}\text { Cour } \\
\text { Suprême }\end{array}$ & $\begin{array}{l}\text { Chambre de la } \\
\text { Cour de cassation }\end{array}$ & $3^{\circ}$ \\
\hline $2^{\circ}$ & Cour d'appel & Cour d'appel & $2^{\circ}$ \\
\hline $1^{\mathrm{o}}$ & $\begin{array}{r}\text { Première } \\
\text { instance }\end{array}$ & $\begin{array}{l}\text { Première } \\
\text { instance }\end{array}$ & $1^{\circ}$ \\
\hline
\end{tabular}

Selon la vision orthodoxe du droit comparé, les juridictions de common law, tels que les Etats-Unis et l'Angleterre, se caractérisent notamment par des décisions judiciaires à grande force obligatoire qui permettent de changer les critères de décision des affaires futures (précédent obligatoire) et leurs décisions comprennent les opinions dissidentes des juges. Les juridictions de civil law comme la France et l'Italie - toujours sous la vision orthodoxe - se caractérisent, en revanche, par des décisions judiciaires à faible force obligatoire, seul un mouvement de plusieurs décisions réunies vers une même fin peut changer les critères de décision des affaires futures (jurisprudence constante) et les décisions ne contiennent pas les opinions dissidentes des juges.

Cette division orthodoxe entre la common law et la civil law est certainement correcte d'un point de vue 'horizontal', c'est-à-dire en comparant des tribunaux situés au 3ème niveau de chaque paire de juridictions : les cours suprêmes des États-Unis et du RoyaumeUni vis-à-vis les chambres en formation ordinaire des cours de cassation en France et en Italie. En effet, les décisions judiciaires des cours suprêmes des États-Unis et du RoyaumeUni ont une grande force obligatoire et les opinions dissidentes sont assez fréquentes. En revanche, les décisions rendues par les chambres en formation ordinaire des cours de cassation en France et en Italie ne profitent pas d'une telle force contraignante et les décisions judiciaires ne contiennent en aucun cas les opinions dissidentes.

Cette division orthodoxe n'est, toutefois, plus valable si l'on passe d'une perspective horizontal à 'diagonale', comparant à présent des tribunaux situés à différents niveaux hiérarchiques. Comme le montre cette thèse à partir d'une comparaison de huit attributions du fonctionnement de ces cours, la France et l'Italie ont également des organes judiciaires avec des fonctions similaires à celles des cours suprêmes des États-Unis et du Royaume-Uni, mais ces organes judiciaires sont, en quelque sorte, 'cachés' à un niveau supérieur. La grande force de pouvoir guider les affaires futures et (de façon rudimentaire) les opinions dissidentes peuvent se trouver non pas dans les chambres en formation ordinaire, mais dans les formations solennelles ou 'formations plénières' des cours de cassation françaises et italiennes. Par conséquent, la fonction judiciaire que l'on trouve dans la common law au 3ème niveau des cours suprêmes des Etats Unis et du Royaume-Uni, est également présente - bien que formulée d'une manière différente et non pas toujours explicitement admise - au 4ème niveau, cachée dans les formations plénières des cours de cassation de France et d'Italie. 
De plus, cette thèse montre que la fonction judiciaire des chambres en formation ordinaire des cours de cassation françaises et italiennes, peut également se retrouver dans les juridictions de common law des Etats Unis et de l'Angleterre, mais à un niveau hiérarchique inférieur dans l'ordre judiciaire. Face à la crise provoquée par une surcharge d'affaires judiciaires, les cours d'appels des Etats-Unis et de l'Angleterre dictent à présent des décisions à force contraignante beaucoup plus faible (comparable au système de jurisprudence constante) et où les opinions dissidentes deviennent une exception peu pratique. Par conséquent, la fonction judiciaire qui est propre à la civil law d'après la

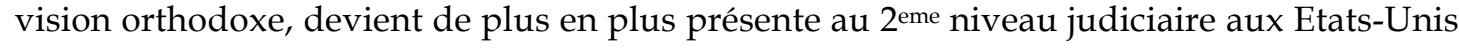
ainsi qu'en Angleterre.

Ces résultats - appelés thèse de la 'symétrie diagonale' - sont important pour plusieurs raisons. Premièrement, la symétrie diagonale nous invite à reformuler la division entre la common law et la civil law. Désormais, la véritable différence ne se trouve pas dans les diverses fonctions judiciaires propres à chaque tradition juridique, mais dans l'ordre hiérarchique judiciaire dans lequel se présente une fonction judiciaire équivalente. Deuxièmement, la symétrie diagonale change la question clé qui devra être répondue à l'avenir par les études de droit comparé. A présent, la question qui se pose n'est pas pourquoi la common law et la civil law ont des fonctions judiciaires différentes, mais pourquoi les juridictions de civil law (comme la France et l'Italie) exercent à un niveau supérieur dans l'ordre judiciaire leurs fonctions, alors que dans les juridictions de common law (tels que les États-Unis et l'Angleterre) elles s'accomplissent par des tribunaux à un niveau inférieur. Troisièmement, la symétrie diagonale a de l'importance pour les acteurs politiques puisqu'elle montre que le point en question lors de l'élaboration d'une réforme judiciaire n'est pas de savoir quelles fonctions devront accomplir les tribunaux, mais à quel niveau du système judiciaire devront-elles être remplies. Enfin, la symétrie diagonale permet la circulation internationale des modèles judiciaires - tels que l'introduction du précédant obligatoire dans les juridictions de civil law - en montrant que les fonctions judiciaires que nous pensions propres à des traditions juridiques étrangères sont, en fait, également présentes dans les juridictions nationales. Ainsi, les soi-disant 'transplantations' de ce type de fonctions judiciaires ne peuvent être vues comme l'introduction d'institutions étrangères, mais comme une relocalisation des fonctions nationales qui étaient auparavant assurées à différents niveaux judiciaires. En ce sens, les résultats de cette thèse seront d'une aide inestimable afin de promouvoir ces avancés dans l'éducation juridique, la recherche comparée et les réformes judiciaires qui se suivent à la thèse de la symétrie diagonale.

\section{Italian}

La presente tesi di dottorato, intitolata "Corti supreme e corti di cassazione: una simmetria diagonale" [Supreme Courts and Courts of Cassation: A Diagonal Symmetry], è uno studio comparatistico delle funzioni giudiziali delle corti di ultima istanza, in campo civile, in due coppie di giurisdizioni che appartengono rispettivamente alla common law (U.S.A. e Inghilterra) e alla civil law (Francia e Italia). La metodologia impiegata è consistita nella comparazione di otto attributi funzionali di tali corti - ovverosia ampiezza del giudizio impugnatorio, effetti delle sentenze, esposizione pubblica, numero dei casi, esame preliminare (o filtri di accesso), composizione del collegio, dimensione totale e stile delle 
sentenze. Ai fini di questa sintesi non possiamo che concentrarci su due di questi attributi funzionali, cioè gli effetti e lo stile delle sentenze. La conclusione principale di questo studio mette in discussione la visione tradizionale sulla distinzione tra civil law e common law per quanto riguarda le (presunte) differenze tra le funzioni giudiziali. Questa ricerca dimostra che, in realtà, entrambi i tipi di giurisdizioni sono dotati di corti con funzioni giudiziali simili, ma situate in posizione differente nella piramide giudiziaria, in questo modo:

Schema I : Sintesi - Una simmetria diagonale

\begin{tabular}{|c|c|c|c|}
\hline & $\begin{array}{r}\text { U.S.A. + } \\
\text { INGHILTERRA }\end{array}$ & $\begin{array}{l}\text { FRANCIA + } \\
\text { ITALIA }\end{array}$ & \\
\hline $4^{\mathrm{o}}$ & & $\begin{array}{l}\text { Sezioni unite della } \\
\text { Cassazione }\end{array}$ & $4^{o}$ \\
\hline $3^{\circ}$ & $\begin{array}{r}\text { Corte } \\
\text { Suprema }\end{array}$ & $\begin{array}{l}\text { Sezione semplice } \\
\text { della Cassazione }\end{array}$ & $3^{\circ}$ \\
\hline $2^{\circ}$ & Corte di appello & Corte di appello & $2^{\circ}$ \\
\hline $1^{\mathrm{o}}$ & Primo grado & Primo grado & $1^{\mathrm{o}}$ \\
\hline
\end{tabular}

Secondo la concezione tradizionale del diritto comparato, le giurisdizioni di common law come gli Stati Uniti o l'Inghilterra si caratterizzano, tra l'altro, perché ciascuna sentenza è dotata di grande forza di cambiamento dei criteri di decisione dei casi successivi (precedente vincolante) e le sentenze contengono le opinioni dissenzienti. Le giurisdizioni di civil law come la Francia e l'Italia - sempre secondo la visione tradizionale - sono, al contrario, caratterizzate da decisioni giudiziali dotate di una forza molto ridotta, poiché solo un indirizzo con molte sentenze convergenti in un' unica direzione potrebbe mutare $\mathrm{i}$ criteri per i casi futuri (giurisprudenza costante) e non è consentito che i giudici esprimano opinioni dissenzienti nelle sentenze.

Questa divisione tradizionale tra common law e civil law risulta certamente corretta secondo una prospettiva "orizzontale", cioè se si comparano corti collocate allo stesso (terzo) livello di ciascuna coppia di giurisdizioni: le corti supreme di Stati Uniti e Regno Unito comparate con le sezioni semplici delle corti di cassazione francese e italiana. Le sentenze delle corti supreme di Stati Uniti e Regno Unito, infatti, hanno una notevole forza vincolante e le opinioni dissenzienti sono piuttosto frequenti. Al contrario, le sentenze emesse le sezioni semplici delle corti di cassazione francese e italiana non godono di tale forza vincolante e nelle loro sentenze è omesso qualsiasi tipo di opinione dissenziente.

Questa stessa suddivisione tradizionale, però, non risulta corretta se mutiamo la nostra prospettiva da orizzontale a "diagonale", comparando tribunali collocati a diversi livelli gerarchici. Come dimostra questa tesi di dottorato, attraverso la comparazione di otto attributi del funzionamento di queste corti, anche la Francia e l'Italia hanno organi giurisdizionali che espletano una funzione simile a quella delle corti supreme degli Stati Uniti e del Regno Unito, ma questi organi giurisdizionali sono, per così dire, "nascosti" a un livello più alto. La grande autorità per guidare la soluzione di casi futuri e (forme rudimentali di) opinioni dissenzienti si possono trovare non nelle sezioni semplici ma 
nelle "adunanze plenarie" delle corti di cassazione francese e italiana. Pertanto, la funzione giudiziale nei contesti di common law, che si trova al terzo livello delle corti supreme degli Stati Uniti e del Regno Unito, è presente anche - per quanto espresso in maniera diversa e non sempre ammesso esplicitamente - nel quarto livello nascosto delle "adunanze plenarie" delle corti di cassazione in Francia e in Italia.

Inoltre, questa tesi di dottorato dimostra che la funzione giudiziale delle sezioni semplici delle corti di cassazione francese e italiana si può ritrovare anche nelle giurisdizioni di common law degli Stati Uniti e dell'Inghilterra, ma a un livello più basso nella piramide giudiziaria. Come reazione alla crisi derivante dal sovraccarico di processi, le corti intermedie di appello negli Stati Uniti e in Inghilterra ora emettono sentenze dotate di una forza vincolante molto più debole (paragonabile al sistema della giurisprudenza costante), in cui le opinioni dissenzienti stanno diventando un'eccezione poco pratica. Pertanto, la funzione giudiziale che, secondo la visione tradizionale, sono proprie della civil law, si stanno rivelando sempre più attuali nel secondo livello delle corti negli Stati Uniti e anche in Inghilterra.

Questi risultati - chiamati la tesi della "simmetria diagonale" - sono rilevanti per diverse ragioni. In primo luogo, la simmetria diagonale ci invita a riformulare la divisione tra common law e civil law. A partire da ora, la vera differenza non è nel diverse funzioni giudiziali in ciascuna tradizione giuridica, ma nel livello gerarchico delle corti in cui una stessa funzione giudiziale è esercitata. In secondo luogo, la simmetria diagonale muta la domanda a cui gli studi di diritto comparato dovranno rispondere in futuro. Da ora, la domanda rilevante non è perché le corti di common law e di civil law hanno funzioni diverse, ma perché le giurisdizioni di civil law (come Francia e Italia) espletano a un livello più alto delle loro corti le funzioni giudiziali che nelle giurisdizioni di common law (come gli Stati Uniti e l'Inghilterra) si compiono a livelli più bassi del sistema giudiziario. In terzo luogo, la simmetria diagonale è importante per gli attori politici perché dimostra che il punto rilevante per disegnare una riforma giudiziale non è quali funzioni si vogliono assegnare alle corti, ma a quale livello del sistema delle corti vogliamo che tali funzioni siano espletate. Infine, la simmetria diagonale potrebbe facilitare la circolazione internazionale dei modelli giudiziali - come l'introduzione del precedente vincolante nelle giurisdizioni di civil law - perché dimostra che le funzioni giudiziali che ritenevamo proprie di tradizioni giuridiche straniere sono, in realtà, presenti anche nelle giurisdizioni nazionali. Pertanto, i cosiddetti "trapianti" di questo tipo di funzioni giudiziali non debbono esser visti come introduzione di istituti stranieri, ma come redistribuzione delle funzioni nazionali che prima erano espletate a diversi livelli del sistema delle corti. In questo senso, i risultati di questa tesi di dottorato saranno di valore inestimabile per promuovere queste migliorie nell'istruzione giuridica, nella ricerca comparatistica e nelle riforme giudiziali che prenderanno le mosse dalla tesi della simmetria diagonale.

\section{Spanish}

Esta tesis doctoral, titulada 'Cortes Supremas y Cortes de Casación: una Simetría Diagonal' [Supreme Courts and Courts of Cassation: A Diagonal Symmetry], es un estudio comparado de las funciones judiciales de las cortes de última instancia en materias civiles en dos pares de jurisdicciones que pertenecen al common law (EEUU e Inglaterra) y al 
civil law o tradición romano-canónica (Francia e Italia). La metodología consistió en la comparación de ocho atributos funcionales de estas cortes - i.e., ámbito de revisión, efectos de las sentencias, exposición pública, número de casos, evaluación preliminar (o filtros de acceso), composición del panel, tamaño total y estilo de las sentencias. Para efectos de este resumen, sin embargo, nos concentraremos en dos de estos atributos, a saber, los efectos y estilo de las sentencias. La conclusión principal de este estudio cuestiona la visión ortodoxa acerca de la división entre el common law y el civil law respecto de las (supuestas) diferencias en sus funciones judiciales. Esta investigación demuestra que, en realidad, ambos pares de jurisdicciones poseen cortes con funciones judiciales similares, pero ubicadas a niveles diferentes en la jerarquía de sus tribunales, del siguiente modo:

Esquema I : Resumen - Una simetría diagonal

\begin{tabular}{|c|c|c|c|}
\hline & $\begin{array}{r}\text { EEUU + } \\
\text { INGLATERRA }\end{array}$ & $\begin{array}{l}\text { FRANCIA + } \\
\text { ITALIA }\end{array}$ & \\
\hline $4^{\mathrm{o}}$ & & $\begin{array}{l}\text { Pleno de } \\
\text { Casación }\end{array}$ & $4^{o}$ \\
\hline $3^{o}$ & $\begin{array}{r}\text { Corte } \\
\text { Suprema }\end{array}$ & $\begin{array}{l}\text { Cámara de } \\
\text { Casación }\end{array}$ & $3^{o}$ \\
\hline $2^{o}$ & $\begin{array}{l}\text { Corte de } \\
\text { Apelación }\end{array}$ & $\begin{array}{l}\text { Corte de } \\
\text { Apelación }\end{array}$ & $2^{o}$ \\
\hline $1^{o}$ & $\begin{array}{l}\text { Primera } \\
\text { Instancia }\end{array}$ & $\begin{array}{l}\text { Primera } \\
\text { Instancia }\end{array}$ & $1^{\mathrm{o}}$ \\
\hline
\end{tabular}

Según la visión ortodoxa en el derecho comparado, las jurisdicciones de common law como EEUU e Inglaterra se caracterizan, entre otros aspectos, porque cada sentencia posee una gran fuerza para cambiar los criterios de decisión de los casos futuros (precedente vinculante) y las sentencias incluyen votos disidentes de los jueces. Las jurisdicciones de civil law como Francia e Italia - continúa la visión ortodoxa - se caracteriza, por el contrario, por decisiones judiciales que poseen una fuerza más débil porque sólo una tendencia de muchas sentencias reunidas en la misma dirección podría cambiar los criterios de los casos futuros (jurisprudencia constante) y que los jueces expresen votos disidentes en las sentencias no está permitido.

Esta división ortodoxa entre common law y civil law ciertamente resulta correcta desde una perspectiva 'horizontal', esto es, cuando se comparan tribunales ubicados en el mismo

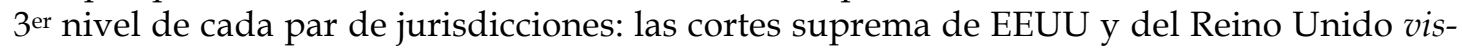
à-vis las cámaras ordinarias de las cortes de casación de Francia e Italia. De hecho, las sentencias de las cortes supremas de EEUU y el Reino Unido tienen una gran fuerza vinculante y los votos disidentes son bastante frecuentes. En cambio, las decisiones dictadas por las cámaras ordinarias de las cortes de casación de Francia e Italia no gozan de tal fuerza vinculante y sus sentencias omiten cualquier tipo de voto disidente.

Esa misma división ortodoxa, sin embargo, no resulta correcta si cambiamos nuestra perspectiva de horizontal a 'diagonal', comparando ahora tribunales ubicados en diferentes niveles jerárquicos. Tal como esta tesis doctoral demuestra a través de la comparación de ocho atributos del funcionamiento de estas cortes, Francia e Italia también 
poseen órganos judiciales que realizan una función similar a las cortes supremas de EEUU y el Reino unido, pero éstos órganos judiciales están, por así decirlo, 'escondidos' en un nivel más alto. La gran fuerza para guiar casos futuros y (formas rudimentarias de) votos disidentes se pueden encontrar no en las cámaras ordinarias sino en las 'sesiones plenarias' de las cortes de casación francesas e italianas. Por lo tanto, la función judicial que en el common law se encuentra en el $3^{\text {er }}$ nivel de las cortes supremas de EEUU y del Reino Unido también está presente - aunque formulado de una manera distinta y no siempre admitido explícitamente - en el $4^{\text {to }}$ nivel escondido de las sesiones plenarias de las cortes de casación en Francia e Italia.

Adicionalmente, esta tesis doctoral demuestra que la función judicial de las cámaras ordinarias de las cortes de casación francesas e italianas también se puede encontrar en las jurisdicciones de common law de EEUU e Inglaterra pero en un nivel más bajo de su jerarquía de los tribunales. Como reacción a la crisis de sobrecarga de casos, las cortes intermedias de apelación en los EEUU e Inglaterra ahora dictan sentencias que tienen una fuerza vinculante mucho más débil (comparable con el sistema de jurisprudencia constante), en las cuales los votos disidentes se están volviendo una excepción poco práctica. Por lo tanto, la función judicial que según la visión ortodoxa es propia del civil law se está volviendo cada vez más presente en el 2do nivel de tribunales en los EEUU e Inglaterra también.

Estos resultados - denominados la tesis de la 'simetría diagonal' - son relevantes por varias razones. Primero, la simetría diagonal nos invita a reformular la división entre el common law y el civil law. A partir de ahora, la verdadera diferencia no está en las distintas funciones judiciales de cada tradición legal, sino en el nivel jerárquico de los tribunales en el cual está presente una función judicial equivalente. Segundo, la simetría diagonal cambia la pregunta relevante que los estudios de derecho comparado deberían contestar a futuro. Desde ahora, la pregunta relevante no es por qué el common law y el civil law poseen funciones judiciales diferentes, sino por qué las jurisdicciones de civil law (como Francia e Italia) cumplen a niveles más altos de sus tribunales las funciones que en las jurisdicciones de common law (como las de EEUU e Inglaterra) se cumplen a niveles más bajos de los tribunales. Tercero, la simetría diagonal es importante para los actores políticos porque demuestra que el punto relevante para diseñar una reforma judicial no es acerca de qué funciones queremos que los tribunales cumplan, sino a qué nivel del sistema de tribunales queremos que esas mismas funciones sean cumplidas. Finalmente, la simetría diagonal facilita la circulación internacional de modelos judiciales - tales como la introducción de precedente vinculante en las jurisdicciones de civil law - porque demuestra que las funciones judiciales que creíamos eran propias de tradiciones jurídicas extranjeras están, en realidad, presentes en las jurisdicciones nacionales también. Por lo tanto, los así llamados 'trasplantes' de este tipo de funciones judiciales no deben ser vistos como la introducción de instituciones extranjeras sino como la reubicación de funciones nacionales que anteriormente eran cumplidas a distintos niveles de los tribunales. En este sentido, los resultados de esta tesis doctoral serán de una ayuda invaluable para promover estas mejoras en la educación jurídica, la investigación comparada y las reformas judiciales que se siguen de la tesis de la simetría diagonal. 


\section{E. Russian}

Данная диссертация «Верховные суды и кассационные суды: диагональная симметрия» - это сравнительное исследование функций судов, являющихся последней инстанцией рассмотрения гражданских дел, в ключе двух парных юрисдикций, принадлежащих общему праву (США и Англия) и системе гражданского права (Франция и Италия). Методологией исследования стало проведение сравнения восьми функциональных признаков этих судов, а именно: объем пересмотра, эффект судебных решений, публичное освещение, количество дел, предварительное разбирательство (критерии доступа), состав коллегии, общее число и способы выражения мнений - но в целях настоящего обзора предлагается сконцентрироваться на эффектах судебных решений и способах выражения мнений. Основные выводы бросают вызов общепринятому взгляду о существующем разделении между гражданским правом и общим правом в отношении (вероятных) различий судебных функций. Данное исследование демонстрирует, что в действительности обе пары юрисдикций имеют суды со схожими функциями, но расположенными на разных уровнях судебной иерархии следующим образом.

\section{Схема 1: Резюме - диагональная симметрия}

\begin{tabular}{|c|c|c|c|}
\hline & США + Англия & \multicolumn{2}{|c|}{ ФРАНЦИЯ + ИТАЛИЯ } \\
\hline $4^{\mathrm{o}}$ & \multirow{3}{*}{$\begin{array}{r}\text { Верховный Суд } \\
\text { Суд } \\
\text { апелляционной } \\
\text { инстанции }\end{array}$} & Кассационный & $4^{\circ}$ \\
\hline $3^{\circ}$ & & $\begin{array}{l}\text { Кассационная } \\
\text { палата }\end{array}$ & $3^{\circ}$ \\
\hline $2^{\circ}$ & & $\begin{array}{l}\text { Суд } \\
\text { апелляционной } \\
\text { инстанции }\end{array}$ & $2^{\circ}$ \\
\hline $1^{\mathrm{o}}$ & $\begin{array}{r}\text { Первая } \\
\text { инстанция }\end{array}$ & $\begin{array}{l}\text { Первая } \\
\text { инстанция }\end{array}$ & $1^{\mathrm{o}}$ \\
\hline
\end{tabular}

Согласно общепринятому в сравнительном правоведении мнению, юрисдикции общего права, такие как США и Англия, кроме прочих признаков характеризуются наличием решений, имеющих влияние на изменение критериев рассмотрения будущих дел (обязательные прецеденты), и судебных решений, включающих особые мнения. Юрисдикции гражданского права, такие как Франция и Италия - в продолжение общепринятой точки зрения, - наоборот характеризуются судебными решениями «слабой силы», поскольку только наличие тенденции - нескольких судебных решений одного направления - может изменить критерии будущих разбирательств (jurisprudence constante [принцип единообразия судебной практики]), и выражение расхожих мнений среди судей не допускается.

Данное традиционное различие между гражданским правом и общим правом, безусловно, справедливо с «горизонтальной» точки зрения - то есть при сравнении 
судов, расположенных на одном третьем уровне каждой пары юрисдикций: Верховные суды США и Великобритании в отношении «кассационных палат» судов Франции и Италии. Действительно, решения верховных судов США и Великобритании имеют строго обязательную силу, и особое мнение встречается довольно часто. С другой стороны, решения, вынесенные обычными палатами французских и итальянских кассационных судов, не имеют эквивалентной обязательной силы и исключают возможность выражения особых мнений.

Однако это различие не имеет места, если изменить перспективу от горизонтального взгляда к «диагонали», сравнивая суды на разных иерархических уровнях. Как показано в диссертации посредством сопоставления восьми признаков работы судов, во Франции и Италии также имеются судебные органы, выполняющие аналогичные функциям верховных судов США и Великобритании действия, но на скрытом, более высоком уровне. Оказание сильного влияния на руководство рассмотрения будущих дел и особые мнения (рудиментарные формы) можно найти не в обычных палатах, а на «пленарных заседаниях» французских и итальянских кассационных судов. Таким образом, функции судов «общего права» найдены на третьем уровне верховных судов США и Великобритании и также присутствуют - однако это сформулировано поразному и не всегда явно признано - в скрытом четвертом уровне кассационного пленума Франции и Италии.

Кроме того, диссертация демонстрирует, что судебные функции обычных палат кассационных судов Франции и Италии также могут быть найдены в юрисдикциях общего права США и Англии, но на более низком судебном уровне. В качестве реакции на кризис, связанный с перегрузкой судов делами, американские и английские промежуточные апелляционные суды теперь выносят решения, где прецедентная сила значительно слабее (в сравнении с системой jurisprudence constante), и в которых особые мнения становятся нецелесообразными и выносятся в исключительных случаях. Таким образом, функции судов, которые согласно общепринятому взгляду присущи системе гражданского права, очевидно, становятся все более применимы на втором уровне судов США и Англии.

Данные выводы - названные тезисом «диагональная симметрия»- актуальны по нескольким причинам. Во-первых, диагональная симметрия предлагает переформулировать разрыв между гражданским и общим правом. Отныне реальный разрыв состоит не в отличиях судебных функций каждой правовой традиции, а в уровне суда, в котором присутствуют эквивалентные функции судебных решений. Вовторых, диагональная симметрия изменяет вопрос, на который должны ответить дальнейшие сравнительно-правовые исследования. Теперь соответствующий вопрос заключается не в том, почему гражданское право и общее право имеют разные функции судов, а почему юрисдикции гражданского права (такие как во Франции и Италии) на высших судебных уровнях осуществляют те функции, которые в юрисдикции общего права (например, США и Англии) представлены в судах более низких уровней. В-третьих, диагональная симметрия важна для политиков, т.к. она демонстрирует соответствующую точку для разработки судебной реформы, касающейся не функций, которые выполнялись бы судами, а уровня судебной системы, на котором требуется выполнение этих функций. В заключение, 
диагональная симметрия может способствовать взаимодействию судебных моделей, например, введению обязательных прецедентов в юрисдикциях гражданского права поскольку это демонстрирует, что судебные функции, которые считаются собственно иностранными юридическими традициями, в действительности также присутствуют и в национальных юрисдикциях. Таким образом, так называемую трансплантацию этих видов судебных функций следует рассматривать не как введение новых иностранных институтов, а как перемещение национальных функций, которые ранее выполнялись на других уровнях суда. В этом смысле результаты данной диссертации станут ценным вкладом в содействии введению этих улучшений в области юридического образования, сравнительных исследований и судебных реформ, которые вытекают из тезиса о диагональной симметрии. 